\title{
Social Inequality in Cigarette Consumption, Cigarette Dependence, and Intention to Quit among Norwegian Smokers
}

\author{
Marianne Lund \\ Norwegian Institute for Alcohol and Drug Research, P.O. Box 565, Centrum, 0105 Oslo, Norway \\ Correspondence should be addressed to Marianne Lund; ml@sirus.no
}

Received 19 December 2014; Revised 13 February 2015; Accepted 26 February 2015

Academic Editor: Mohammed Jawad

Copyright ( 2015 Marianne Lund. This is an open access article distributed under the Creative Commons Attribution License, which permits unrestricted use, distribution, and reproduction in any medium, provided the original work is properly cited.

\begin{abstract}
Background. The study aim was to examine the influence of education and income on multiple measures of risk of smoking continuation. Methods. Three logistic regression models were run on cigarette consumption, dependence, and intention to quit based on nationally representative samples (2007-2012) of approximately 1200 current smokers aged 30-66 years in Norway. Results. The relative risk ratio for current versus never smokers was RRR 5.37, 95\% CI [4.26-6.77] among individuals with low educational level versus high and RRR 1.53, 95\% CI [1.14-2.06] in the low-income group versus high (adjusted model). Low educational level was associated with high cigarette consumption, high cigarette dependence, and no intention to quit. The difference in predicted probability for having high cigarette consumption, high cigarette dependence, and no intention to quit were in the range of 10-20 percentage points between smokers with low versus those with high educational level. A significant difference between low- and high-income levels was observed for intention to quit. The effect of education on high consumption and dependence was mainly found in smokers with high income. Conclusion. Increased effort to combat social differences in smoking behaviour is needed. Implementation of smoking cessation programmes with high reach among low socioeconomic groups is recommended.
\end{abstract}

\section{Introduction}

While smoking rates among countries across the Western world are gradually decreasing, concerns over social inequality in smoking behaviour are increasing. Many studies have found an association between smoking behaviour and different measures of socioeconomic status (SES) such as education, income, and occupational class [1-3]. Smokers with low SES also have poorer cessation outcomes. This inequality pattern has been observed in studies of smoking cessation interventions and aggregated-level quit rates [4-6]. There is also some evidence of increasing social inequality in smoking behaviour and substantial health disparity consequences $[7$, 8].

In Norway, smoking rates are gradually declining, with a rate of $22 \%$ in 2014 in the adult population aged $16-74$ years (13\% are daily smokers). Norway has a strong welfare system and strives to be an egalitarian society that provides equal opportunities for all citizens. Despite reduced mortality in all educational groups, educational inequality in mortality increased in Norway in the period 1960-2000 [9]. Smoking is one mechanism behind this inequality [10]. Increased knowledge about social inequality in smoking behaviour can inform tobacco prevention efforts.

The pathways to successful quitting have been widely studied. Nicotine dependence is regarded as a primary barrier to giving up smoking and is predictive of smoking continuation [11]. Number of cigarettes per day (CPD) has often been used as a proxy for nicotine dependence, although some studies indicate that one should be cautious in interpreting high cigarette consumption as nicotine dependence. CPD is significantly associated with nicotine dependence, but differences in dependence are found to be independent of CPD level [12]. However, high cigarette consumption indicates a strong habit and illustrates aspects of dependence such as the time and effort the smoker dedicate to the behaviour [13].

Nicotine dependence has been widely measured in population-based surveys using different measurements like the Fagerström Test for Nicotine Dependence (FTND) and associated short versions such as the Heaviness of Smoking Index (HSI) and time to first cigarette in the morning (TTFC). The TTFC is likely the single item in the FTND 
that most strongly predicts addiction to nicotine, probably because morning smoking reflects the smoker's overnight withdrawal symptoms [14]. TTFC also shows good correlation with biological measures of nicotine ingestion [15].

The FTND, TTFC, and number of CPD are all predictive of smoking continuation and significantly associated with relapse following a quit attempt $[16,17]$. Having an intention to quit smoking is strongly associated with quit attempts but is less consistent with quitting success [18].

Measures of nicotine dependence such as the FTND, HSI, and TTFC are significantly related to SES and show increasing dependence with decreasing SES $[17,19,20]$. The association between nicotine dependence and SES is also found in studies using biochemical measures of dependence, such as levels of cotinine in plasma [21].

However, the association between intention to quit and SES is less clear. Some studies report a positive relationship between low-SES smokers and intention to quit or quit attempts but reduced smoking cessation success among lowSES smokers $[6,22]$. Other studies investigating the transtheoretical model of change report a higher proportion of smokers with a low educational level in the precontemplation stage (i.e., a smoker who does not intend to quit) $[23,24]$.

Norway is in the final stage of the tobacco epidemic, experiencing both a gradual decline in smoking prevalence and persistent inequality in smoking habits. In this situation, it is of interest to investigate differences in smoking behaviour that indicate smoking continuation. Risk of continued smoking is defined in three ways: high cigarette consumption, high cigarette dependence, and having no intention to quit. The aim of this study was to investigate the associations between education and income and risk of high cigarette consumption, cigarette dependence, and intention to quit. Because education and income are related, it was of interest to explore the combined effect of social inequality measures on risk of smoking continuation.

\section{Method}

2.1. Study Sample. Data were pooled from six cross-sectional datasets representative of the Norwegian population during 2007-2012. Approximately 1200 respondents aged 16 years or older were surveyed by telephone during the autumn of each year by Statistics Norway. The study sample was 4591 respondents aged 30-66 years. The lower age cut-off for inclusion was 30 years because younger adults may not have completed their education. A study sample aged 30 years or older also represents a population of individuals with an established smoking history, since more than half of daily smokers start smoking before age 18. Individuals who received early retirement pensions $(n=197)$ were excluded from the study sample, along with 89 individuals with missing education information. Survey response rates were 67\% (2007), 57\% (2008), 61\% (2009), 54\% (2010), 58\% (2011), and 61\% (2012).

2.2. Dependent Variables: Cigarette Consumption, Cigarette Dependence, and Absence of Intention to Quit. Three measures were used to capture risk of smoking continuation: cigarette consumption, nicotine dependence, and intention to quit. High cigarette consumption was defined as consumption of $15 \mathrm{CPD}$ or more. Occasional smokers with an average weekly consumption above 105 were coded in the +15 CPD group. Cigarette dependence was the time to first cigarette in the morning (TTFC); individuals smoking within the first 30 minutes after awakening were defined as having high cigarette dependence and individuals who smoked 31 minutes or more after wakening had low cigarette dependence [14]. Although TTFC is most often referred to as a measure of nicotine dependence, it also captures nonpharmacological aspects of cigarette dependence such as psychosocial functions [13]. The term cigarette dependence is therefore preferred in the present study. Having no intention to quit was a measure of smokers' short- or long-term intention to quit; smokers with no intention to quit within the next 6 months and who also believed they would still be smoking in 5 years were defined as having no intention to quit.

2.3. Socioeconomic Measures: Education and Income. Two measures of SES were included as independent variables: educational level and income level. Educational level was recoded from the original nine-level variable to three levels: completion of lower secondary, upper secondary, and university levels. For the interaction analysis, we used a dichotomous measure of education with high educational level including completion of upper secondary school or university and low educational level representing completion of lower secondary school. Income was defined by combining the gross household income and marital status. Those with an annual household income above the median (NOK 700000 , $\approx$ USD 160000 or more) were coded in the high-income group. Medium income was NOK $300000-699000(\approx$ USD 36000-50 000) and low income was below NOK 300000. Those with a household income of NOK 300 000-699000 and living alone were coded as having high income. In the study sample, $12 \%$ were in the low-income group (7\% of the population sample, see Table 1). This is comparable to the percentage defined as having low income in Norway using the EU definition of $60 \%$ of median income [25].

2.4. Analyses. Data analyses were conducted in two parts. First, the representative sample was used to confirm socioeconomic differences in smoking status. For the multinomial regression, the smoking outcome category was defined as current and former smokers, with nonsmokers as the reference category. Results from this analysis are presented as a relative risk ratio (RRR) in Table 2 . The characteristics of the population sample and study sample of all current smokers (daily and occasional smokers) are presented in Table 1. The logistic regression analysis included three binary outcomes reflecting risk of smoking continuation (cigarette consumption, cigarette dependence, and intention to quit), with education and income as independent variables. The models were adjusted for survey year, age, sex, and numbers of household members. Three logistic models were used to compute adjusted prediction (predicted probabilities) of the 
TABLE 1: Characteristics of the population and study samples (current smokers). Participants aged 30-66 years. Data were pooled from 2007 to 2012 .

\begin{tabular}{|c|c|c|c|c|}
\hline & $\begin{array}{l}\text { Population sample } \\
\qquad(N=4600)\end{array}$ & $n$ & $\begin{array}{c}\text { Study sample } \\
\text { (current smokers, } n=1282 \text { ) }\end{array}$ & $n$ \\
\hline Age (mean, SD) & $47.7(10.2)$ & 4600 & $47.7(9.7)$ & 1282 \\
\hline Male $(\%)$ & 49.1 & 2260 & 49.9 & 640 \\
\hline \multicolumn{5}{|l|}{ Educational level } \\
\hline High & 39.1 & 1798 & 23.7 & 304 \\
\hline Medium & 43.7 & 2008 & 47.4 & 607 \\
\hline Low & 17.3 & 794 & 28.9 & 371 \\
\hline \multicolumn{5}{|l|}{ Household income } \\
\hline High & 66.9 & 2849 & 60.1 & 701 \\
\hline Medium & 25.9 & 1105 & 27.8 & 324 \\
\hline Low & 7.2 & 308 & 12.2 & 142 \\
\hline Daily smokers (\%) & 20.4 & 937 & 73.1 & 937 \\
\hline Heavy smoking $\geq 15 \mathrm{CPD}$ & 7.9 & 365 & 28.5 & 365 \\
\hline TTFC $\leq 30$ minutes & 9.3 & 423 & 34.3 & 423 \\
\hline No intention to quit & 7.1 & 327 & 25.7 & 237 \\
\hline
\end{tabular}

TABLE 2: Adjusted multinomial regression for education and income according to smoking status with never smoker as reference group. Relative risk ratio (RRR) and 95\% confidence interval. Bivariate and adjusted models.

\begin{tabular}{|c|c|c|c|c|}
\hline & \multicolumn{2}{|c|}{$\begin{array}{l}\text { Model 1: bivariate relationship } \\
\quad \text { Never smoker = ref. }\end{array}$} & \multicolumn{2}{|c|}{$\begin{array}{c}\text { Model 2: adjusted for survey year, age, sex, and } \\
\text { members of the household } \\
\text { Never smoker }=\text { ref. }\end{array}$} \\
\hline & Current smoker & Former smoker & Current smoker & Former smoker \\
\hline High education & Ref. & Ref. & Ref. & Ref. \\
\hline Medium education & $2.59(2.21,3.06)^{* * *}$ & $1.92(1.63,2.25)^{* * *}$ & $2.53(2.12,3.02)^{* * *}$ & $1.75(1.47,2.08)^{* * *}$ \\
\hline Low education & $5.66(4.61,6.95)^{* * *}$ & $2.31(1.84,2.89)^{* * *}$ & $5.37(4.26,6.77)^{* * *}$ & $2.05(1.59,2.65)^{* * *}$ \\
\hline High income & Ref. & Ref. & Ref. & Ref. \\
\hline Medium income & $1.44(1.22,1.70)^{* * *}$ & $1.44(1.22,1.71)^{* * *}$ & $1.07(0.89,1.29)$ & $1.16(0.97,1.40)$ \\
\hline Low income & $2.76(2.12,3.59)^{* * *}$ & $1.17(0.84,1.64)$ & $1.53(1.14,2.06)^{* *}$ & $0.84(0.58,1.21)$ \\
\hline
\end{tabular}

${ }^{* * *} P<.001,{ }^{* *} P<.01,{ }^{*} P<.05$.

outcomes across the SES measures and marginal effects (differences in predicted probabilities) between different levels of SES (Table 3). Marginal effects show how the outcome changed for each change in the categorical independent variable. Marginal effects are estimated as average marginal effects, which means that other variables in the model are used as observed for each case. Tables 4 show the predicted probability for each combined group of education and income using the margins command (education \# income) and the delta method was used to examine the statistical significance of group comparisons [26]. Only dichotomous measures of education and income were used for the combined effect (high versus low). All analyses were conducted using Stata statistical software (v.13).

\section{Results}

The proportion of individuals with low educational level (28.9\%) was higher in the study sample of current smokers than in the population sample (17.3\%) (Table 1). The proportion with low income level was $12.2 \%$ in the study sample and $7.2 \%$ in the population sample. One out of four current smokers reported having high cigarette consumption and no intention to quit, while one out of three reported having high cigarette dependence. Social inequality in smoking behaviour was confirmed. Educational differences were present in both the bivariate and the adjusted models, with RRR of 5.37, 95\% confidence interval [4.26-6.77] for current compared with never smokers in the low educational level (Table 2). 
TABLE 3: Adjusted predicted probabilities and marginal effects (differences in predicted probabilities) of the outcomes high consumption, high cigarette dependence, and no intention to quit smoking by education and income. All variables included in each model, in addition to survey year, age, sex, and number of persons in household. Current smokers aged 30-66 years. Data were pooled from 2007 to 2012.

\begin{tabular}{|c|c|c|c|c|c|c|}
\hline & \multicolumn{2}{|c|}{$\begin{array}{l}\text { High consumption } \\
\qquad N=1147 \\
\text { Percent }(95 \% \mathrm{CI})\end{array}$} & \multicolumn{2}{|c|}{$\begin{array}{l}\text { High cigarette dependence } \\
\qquad N=1105 \\
\text { Percent }(95 \% \mathrm{CI})\end{array}$} & \multicolumn{2}{|c|}{$\begin{array}{l}\text { No intention to quit } \\
\qquad N=1142 \\
\text { Percent }(95 \% \mathrm{CI})\end{array}$} \\
\hline & $\begin{array}{l}\text { Adjusted } \\
\text { predicted } \\
\text { probability }\end{array}$ & $\begin{array}{l}\text { Marginal effects } \\
\text { (difference in } \\
\text { predicted } \\
\text { probability) }\end{array}$ & $\begin{array}{l}\text { Adjusted } \\
\text { predicted } \\
\text { probability }\end{array}$ & $\begin{array}{l}\text { Marginal effects } \\
\text { (difference in } \\
\text { predicted } \\
\text { probability) }\end{array}$ & $\begin{array}{l}\text { Adjusted } \\
\text { predicted } \\
\text { probability }\end{array}$ & $\begin{array}{l}\text { Marginal effects } \\
\text { (difference in } \\
\text { predicted } \\
\text { probability) }\end{array}$ \\
\hline \multicolumn{7}{|l|}{ Education } \\
\hline High & $17.9(13.2,22.6)$ & Reference & $19.8(14.7,24.8)$ & Reference & $19.1(14.2,24.1)$ & Reference \\
\hline Medium & $29.8(26.1,33.5)$ & $11.9(5.9,18.0)^{* * *}$ & $36.3(32.2,40.3)$ & $16.5(10.0,23.0)^{* * *}$ & $25.0(21.4,28.5)$ & $5.9(-0.2,12.0)$ \\
\hline Low & $33.3(28.1,38.4)$ & $15.4(8.2,22.5)^{* * *}$ & $39.0(33.5,44.4)$ & $19.2(11.6,26.8)^{* * *}$ & $30.6(25.6,35.6)$ & $11.5(4.3,18.7)^{* *}$ \\
\hline \multicolumn{7}{|l|}{ Income } \\
\hline High & $25.1(21.8,28.4)$ & Reference & $29.5(25.9,33.1)$ & Reference & $20.8(17.9,24.3)$ & Reference \\
\hline Medium & $35.1(29.8,40.3)$ & $10.1(3.7,16.3)^{* *}$ & $39.3(33.9,44.7)$ & $9.8(3.2,16.4)^{* *}$ & $30.4(24.8,35.0)$ & $9.6(3.5,15.7)^{* *}$ \\
\hline Low & $26.2(19.0,33.4)$ & $1.1(-7.0,9.2)$ & $36.6(28.4,44.8)$ & $7.1(-2.0,16.2)$ & $34.7(25.1,42.1)$ & $13.9(4.8,23.1)^{* *}$ \\
\hline
\end{tabular}

TABLE 4: Margins (adjusted predicted probability) for high consumption of cigarettes, high cigarette dependence, and no intention to quit by education and income (margins income \# education).

\begin{tabular}{|c|c|c|c|c|c|c|c|c|c|c|}
\hline \multirow{2}{*}{ Education } & \multirow{2}{*}{ Income } & \multicolumn{2}{|c|}{ High cigarette consumption } & \multirow[t]{2}{*}{$n$} & \multicolumn{2}{|c|}{ High cigarette dependence } & \multirow[t]{2}{*}{$n$} & \multicolumn{2}{|c|}{ No intention to quit } & \multirow[t]{2}{*}{$n$} \\
\hline & & Margins & Unadjusted groups & & Margins & Unadjusted groups & & Margins & Unadjusted groups & \\
\hline High & High & 25.7 & A & 758 & 30.3 & A & 725 & 21.4 & A & 755 \\
\hline High & Low & 26.7 & A & 76 & 34.2 & A & 74 & 31.8 & A & 76 \\
\hline Low & High & 35.9 & B & 252 & 39.7 & B & 247 & 30.6 & B & 252 \\
\hline Low & Low & 26.2 & $\mathrm{~A}$ & 61 & 40.9 & $\mathrm{~A}$ & 59 & 40.3 & $\mathrm{~B}$ & 59 \\
\hline
\end{tabular}

Margins sharing a letter in the group label are not significantly different at the $5 \%$ level.

A significant association between income and current smoking was observed in the bivariate model. In the adjusted model, the RRR for current smoking was 1.53 , 95\% confidence interval [1.14-2.06] in the lowest compared with the highest income group.

Table 3 presents three separate logistic regression models for the outcome variables high cigarette consumption, high cigarette dependence, and having no intention to quit. Adjusted predicted probabilities for the outcomes of interest are presented for each SES group. Among current smokers, the probability of high cigarette consumption, high cigarette dependence, and having no intention to quit increased with reduced educational level (Table 3 ). The marginal effect shows a 15 percentage point increase between the highest and lowest educational groups in the predicted probability of having high cigarette consumption. The marginal effect of education on cigarette dependence showed a 19 percentage point increase.

Income produced somewhat different results than educational level, with the highest probability of the outcomes of high consumption and high cigarette dependence among those with medium income level. Low-income smokers had the same probability of being a high-consuming smoker as the high-income group, 26\% and 25\%, respectively (Table 3 ). The probability for cigarette dependence for high-, medium-, and low-income groups was 30\%, 39\%, and 37\%, respectively.

Having no intention to quit was significantly associated with low educational level and low or medium income (Table 3). The adjusted predicted probability that a smoker with a low educational level would have no intention to quit was $31 \%$, while the corresponding percentage for smokers with a high educational level was $19 \%$.

Table 4 presents the adjusted predicted probabilities for the outcome variables for every combination of high and low educational levels and income. The education effect for the outcome cigarette consumption and cigarette dependence was only found among those with high income. There was a 10 percentage point difference in the probability of having a high cigarette consumption and being highly dependent on cigarettes between the highly educated with high income compared with those with a low educational level with high income (Table 4). A 10 percentage point difference was also found for cigarette dependence between those with high levels of both education and income compared with those with low levels of both education and income ("top-bottom" differences), but the difference did not reach statistical significance. An educational effect among the high-income smokers was 
also found for no intention to quit smoking, with a 9 percentage point difference. A significant "top-bottom" difference for having no intention to quit smoking was also observed, with a 19 percentage point difference in predicted probabilities. For example, a smoker with high educational level and high income had a predicted $21 \%$ chance of having no intention to quit smoking, while the corresponding number for a smoker with low educational level and low income was $40 \%$.

\section{Discussion}

This study revealed a strong association between education and the outcomes indicating risk of smoking continuation: high cigarette consumption, high cigarette dependence, and having no intention to quit. Low income had an independent effect on intention to quit. The effect of education was only valid for those defined as having a high-income level. There was a 10-20 percentage point difference between high and low education level in relation to probability of high consumption, dependence, and no intention to quit.

Several studies confirm the importance of education for lack of smoking cessation and risk of smoking continuation $[5,19,21,27]$. Possible explanations for the strong influence of education on smoking have included knowledge and cognitive resources, social networks, number of smokers and social norms regarding smoking in the social environment, health literacy, psychosocial stress, and health risk perceptions [28-30]. It has been suggested that education creates a culture that discourages smoking [31]. Being in a culture where smokers are in the minority and where norms against smoking dominate may make it easier for someone who smokes to quit. Stronger no-smoking norms among those with greater education may explain some of their lower risk of smoking continuation.

The strong association between education and smoking continuation may be ascribed to the association between delay discounting/impulsivity and education; several studies show that less educated individuals choose smaller, immediate rewards over larger, delayed rewards $[32,33]$. This means that smoking would be valued more highly than future health. Current smokers discount delayed rewards more than never and former smokers and are more nicotine dependent than less dependent smokers, even when controlling for education $[34,35]$. However, the association between education/income and nicotine dependence is stronger than the association between delay discounting and nicotine dependence [35]. A Norwegian study of adolescents found that both education and impulsivity predicted smoking initiation, but only education (not impulsivity) predicted smoking cessation. No interaction between education and impulsivity on smoking cessation was found [36].

The somewhat stronger relationship between education and smoking behaviour compared with income and smoking behaviour may vary by country [37]. Income had a curvilinear impact on high consumption. The high price of cigarettes in Norway may explain the low probability of high cigarette consumption in the low-income group, a finding in line with studies showing that low-SES groups are sensitive to increasing cigarette taxes [38]. However, this does not explain the low consumption levels among the highincome group in this study. Having low income may reduce cigarette consumption, but being financially deprived does not necessary imply an increased motivation to quit smoking.

Increases in the price of or tax on cigarettes are seen as having the most consistent positive impact, for example, the greatest potential to reduce inequality in smoking behaviour [38]. Interventions such as compulsory and national smokefree policies and control on advertising, promotion and marketing of tobacco are regarded as having a positive or neutral impact; here, a neutral impact means that the effect would be equal regarding SES [38]. Norway scores relatively high on the cigarettes price score ( 20 out of 30 points) in the tobacco control scale in Europe [39]. Further tax increases are seen as problematic due to fear of increased cross-border trade with subsequent lost tax revenue and smuggling. Smoke-free legislation was introduced in Norway in 2004, with positive health effects among employees in the hospitality industry [40]. The impact of national smoke-free policies on reducing inequalities is found mainly in reduced social inequalities in passive smoking (nine out of 19 studies) [38]. Smoke-free legislation is expected to reduce the social acceptability of smoking, thereby contributing to the ongoing process of smoking denormalization. Whether denormalization processes have the same impact regardless of social status is unclear and highly debated (cf. the smoker stigma debate [41]).

Tobacco control interventions such as price/taxation increases and sales restrictions are considered highly effective because they affect most people. The population-level cessation support in Norway, with the exception of individual media campaigns that have been launched earlier, comprises a national quit line and a web site for smoking cessation support hosted by health authorities. Call rates to the quit line are higher among high-SES groups than low-SES groups and these SES differences are stable over time [42]. A study evaluating the Norwegian quit line is currently in progress. More intensive smoking cessation services implemented through the health care service with special focus on deprived areas have shown positive effects in reducing social inequality in smoking in England [43]. Reaching proportionally more low-SES smokers than high-SES smokers may compensate for the lower quit rates usually found in socially disadvantaged groups of smokers.

The present study results are consistent with others and show the need to increase motivation to quit and assist nicotine-dependent low-SES smokers to quit smoking. In addition, the present study has disentangled the effect of two SES measures (education and income) on three separate indicators of prolonged smoking. The results show substantial differences in motivation to quit between those with both high educational level and high income, compared with those with both low educational level and low income.

Many Western countries including Norway have made substantial progress in reducing smoking prevalence over the last two decades but have been unable to decrease social inequality in smoking behaviour. New population-based interventions are currently being debated, including plain packaging and harm reduction strategies such as use of electronic cigarettes. Given the high mortality rate from cigarette 
smoking and its contribution to health inequality, interventions that reduce smoking rates in low-SES populations are needed. However, few population-based interventions with an equity impact beyond those already identified, including price and tax increases, exist. A report from the Royal College of Physicians states that harm reduction strategies, such as electronic cigarettes, may have a potential role in preventing deaths from cigarette smoking and reducing social inequalities in smoking-related morbidity and mortality [44]. Further investigation on the potential role of electronic cigarettes to reduce social inequality in smoking is needed, both to assess their potential for helping nicotine-dependent smokers to quit as well as their potential to increase motivation to quit among smokers unwilling to quit smoking.

\section{Limitations}

The cross-sectional design of this study makes it impossible to deduce causation. The validity of the outcome variables requires attention. Having high cigarette consumption, high cigarette dependence, and no intention to quit were used as indices of risk for smoking continuation. This is consistent with several studies reporting these measures in relation to unsuccessful cessation among hardcore smokers. In a longitudinal study, the predictive ability of high consumption, high dependence, and intention to quit was investigated in relation to continued smoking after 1 year. All components predicted smoking continuation, but nicotine dependence was the best predictor of smoking continuation [16].

\section{Conflict of Interests}

No conflict of interests exists.

\section{References}

[1] E. M. Barbeau, N. Krieger, and M.-J. Soobader, "Working class matters: socioeconomic disadvantage, race/ethnicity, gender, and smoking in NHIS 2000," American Journal of Public Health, vol. 94, no. 2, pp. 269-278, 2004.

[2] M. M. Schaap, H. M. van Agt, and A. E. Kunst, "Identification of socioeconomic groups at increased risk for smoking in European countries: looking beyond educational level," Nicotine \& Tobacco Research, vol. 10, no. 2, pp. 359-369, 2008.

[3] J. L. Reid, D. P. Hammond, and P. M. Driezen, "Socio-economic status and smoking in Canada, 1999-2006: Has there been any progress on disparities in Tobacco use?" Canadian Journal of Public Health, vol. 101, no. 1, pp. 73-78, 2010.

[4] R. Hiscock, K. Judge, and L. Bauld, "Social inequalities in quitting smoking: what factors mediate the relationship between socioeconomic position and smoking cessation?" Journal of Public Health, vol. 33, no. 1, pp. 39-47, 2011.

[5] B. Federico, G. Costa, and A. E. Kunst, "Educational inequalities in initiation, cessation, and prevalence of smoking among 3 Italian birth cohorts," American Journal of Public Health, vol. 97, no. 5, pp. 838-845, 2007.

[6] D. Kotz and R. West, "Explaining the social gradient in smoking cessation: it's not in the trying, but in the succeeding," Tobacco Control, vol. 18, no. 1, pp. 43-46, 2009.
[7] M. de Looze, T. ter Bogt, A. Hublet et al., “Trends in educational differences in adolescent daily smoking across Europe, 200210," The European Journal of Public Health, vol. 23, no. 5, pp. 846-852, 2013.

[8] K. Giskes, A. E. Kunst, J. Benach et al., "Trends in smoking behaviour between 1985 and 2000 in nine European countries by education," Journal of Epidemiology and Community Health, vol. 59, no. 5, pp. 395-401, 2005.

[9] B. H. Strand, E.-K. Grøholt, O. A. Steingrímsdottír, T. Blakely, S. Graff-Iversen, and $\varnothing$. Næss, "Educational inequalities in mortality over four decades in Norway: prospective study of middle aged men and women followed for cause specific mortality, 1960-2000," British Medical Journal, vol. 340, article c654, 2010.

[10] T. A. Eikemo, R. Hoffmann, M. C. Kulik et al., "How can inequalities in mortality be reduced? A quantitative analysis of 6 risk factors in 21 european populations," PLoS ONE, vol. 9, no. 11, Article ID el10952, 2014.

[11] N. Breslau, E. O. Johnson, E. Hiripi, and R. Kessler, "Nicotine dependence in the United States: prevalence, trends, and smoking persistence," Archives of General Psychiatry, vol. 58, no. 9, pp. 810-816, 2001.

[12] E. C. Donny and L. C. Dierker, "The absence of DSM-IV nicotine dependence in moderate-to-heavy daily smokers," Drug and Alcohol Dependence, vol. 89, no. 1, pp. 93-96, 2007.

[13] K. Fagerström, "Determinants of tobacco use and renaming the FTND to the Fagerström test for cigarette dependence," Nicotine \& Tobacco Research, vol. 14, no. 1, Article ID ntr137, pp. 75-78, 2012.

[14] S. A. Branstetter and J. E. Muscat, "Time to first cigarette and serum cotinine levels in adolescent smokers: national health and nutrition examination survey, 2007-2010," Nicotine and Tobacco Research, vol. 15, no. 3, pp. 701-707, 2013.

[15] J. E. Muscat, S. D. Stellman, R. S. Caraballo, and J. P. Richie Jr., "Time to first cigarette after waking predicts cotinine levels," Cancer Epidemiology Biomarkers and Prevention, vol. 18, no. 12, pp. 3415-3420, 2009.

[16] D. T. Ip, J. E. Cohen, S. J. Bondy et al., "Do components of current 'hardcore smoker' definitions predict quitting behaviour?" Addiction, vol. 107, no. 2, pp. 434-440, 2012.

[17] J. A. Fidler, L. Shahab, and R. West, "Strength of urges to smoke as a measure of severity of cigarette dependence: comparison with the Fagerström Test for Nicotine Dependence and its components," Addiction, vol. 106, no. 3, pp. 631-638, 2011.

[18] E. Vangeli, J. Stapleton, E. S. Smit, R. Borland, and R. West, "Predictors of attempts to stop smoking and their success in adult general population samples: a systematic review," Addiction, vol. 106, no. 12, pp. 2110-2121, 2011.

[19] M. Siahpush, A. McNeill, R. Borland, and G. T. Fong, "Socioeconomic variations in nicotine dependence, self-efficacy, and intention to quit across four countries: findings from the International Tobacco Control (ITC) Four Country Survey," Tobacco Control, vol. 15, no. 3, pp. iii71-iii75, 2006.

[20] G. Docherty, A. Mcneill, C. Gartner, and L. Szatkowski, "Did hardening occur among smokers in England from 2000 to 2010?" Addiction, vol. 109, no. 1, pp. 147-154, 2014.

[21] M. Pennanen, U. Broms, T. Korhonen et al., "Smoking, nicotine dependence and nicotine intake by socio-economic status and marital status," Addictive Behaviors, vol. 39, no. 7, pp. 1145-1151, 2014.

[22] M. Siahpush, H.-H. Yong, R. Borland, J. L. Reid, and D. Hammond, "Smokers with financial stress are more likely to 
want to quit but less likely to try or succeed: findings from the International Tobacco Control (ITC) Four Country Survey," Addiction, vol. 104, no. 8, pp. 1382-1390, 2009.

[23] U. John, C. Meyer, H.-J. Rumpf, and U. Hapke, "Relation among stage of change, demographic characteristics, smoking history, and nicotine dependence in an adult German population," Preventive Medicine, vol. 37, no. 4, pp. 368-374, 2003.

[24] M. E. Wewers, F. A. Stillman, A. M. Hartman, and D. R. Shopland, "Distribution of daily smokers by stage of change: current population survey results," Preventive Medicine, vol. 36, no. 6, pp. 710-720, 2003.

[25] Statistics Norway, Households income, 2014, http://www.ssb .no/en/inntind.

[26] R. Williams, "Using the margins command to estimate and interpret adjusted predictions and marginal effects," The Stata Journal, vol. 12, no. 2, pp. 308-331, 2012.

[27] R. A. Schnoll, A. Goren, K. Annunziata, and J. A. Suaya, "The prevalence, predictors and associated health outcomes of high nicotine dependence using three measures among US smokers," Addiction, vol. 108, no. 11, pp. 1989-2000, 2013.

[28] R. Hiscock, L. Bauld, A. Amos, J. A. Fidler, and M. Munafò, "Socioeconomic status and smoking: a review," Annals of the New York Academy of Sciences, vol. 1248, no. 1, pp. 107-123, 2012.

[29] D. W. Stewart, C. E. Adams, M. A. Cano et al., "Associations between health literacy and established predictors of smoking cessation," American Journal of Public Health, vol. 103, no. 7, pp. e43-e49, 2013.

[30] N. Slopen, E. Z. Kontos, C. D. Ryff, J. Z. Ayanian, M. A. Albert, and D. R. Williams, "Psychosocial stress and cigarette smoking persistence, cessation, and relapse over 9-10 years: a prospective study of middle-aged adults in the United States," Cancer Causes \& Control, vol. 24, no. 10, pp. 1849-1863, 2013.

[31] W. Johnson, K. O. Kyvik, E. L. Mortensen, A. Skytthe, G. D. Batty, and I. J. Deary, "Does education confer a culture of healthy behavior? Smoking and drinking patterns in danish twins," American Journal of Epidemiology, vol. 173, no. 1, pp. 5563, 2011.

[32] J. L. Jaroni, S. M. Wright, C. Lerman, and L. H. Epstein, "Relationship between education and delay discounting in smokers," Addictive Behaviors, vol. 29, no. 6, pp. 1171-1175, 2004.

[33] S. Reimers, E. A. Maylor, N. Stewart, and N. Chater, "Associations between a one-shot delay discounting measure and age, income, education and real-world impulsive behavior," Personality and Individual Differences, vol. 47, no. 8, pp. 973978, 2009.

[34] M. M. Sweitzer, E. C. Donny, L. C. Dierker, J. D. Flory, and S. B. Manuck, "Delay discounting and smoking: association with the Fagerström Test for Nicotine Dependence but not cigarettes smoked per day," Nicotine \& Tobacco Research, vol. 10, no. 10, pp. 1571-1575, 2008.

[35] M. Amlung and J. MacKillop, "Clarifying the relationship between impulsive delay discounting and nicotine dependence," Psychology of Addictive Behaviors, vol. 28, no. 3, pp. 761-768, 2014.

[36] E. Kvaavik and J. Rise, "How do impulsivity and education relate to smoking initiation and cessation among young adults?" Journal of Studies on Alcohol and Drugs, vol. 73, no. 5, pp. 804810, 2012.

[37] M. Leinsalu, C. Kaposávri, and A. E. Kunst, "Is income or employment a stronger predictor of smoking than education in economically less developed countries? A cross-sectional study in Hungary," BMC Public Health, vol. 11, no. 1, article 97, 2011.
[38] T. Brown, S. Platt, and A. Amos, "Equity impact of European individual-level smoking cessation interventions to reduce smoking in adults: a systematic review," The European Journal of Public Health, vol. 24, no. 4, pp. 551-556, 2014.

[39] L. Joossens and M. Raw, "The tobacco control scale 2013 in Europe," A Report of the Association of European Cancer Leagues, 2013.

[40] T. M. L. Eagan, J. Hetland, and L. E. Aarø, "Decline in respiratory symptoms in service workers five months after a public smoking ban," Tobacco Control, vol. 15, no. 3, pp. 242246, 2006.

[41] J. Stuber, I. Meyer, and B. Link, "Stigma, prejudice, discrimination and health," Social Science \& Medicine, vol. 67, no. 3, pp. 351-357, 2008.

[42] M. Siahpush, M. Wakefield, M. Spittal, and S. Durkin, "Antismoking television advertising and socioeconomic variations in calls to Quitline," Journal of Epidemiology and Community Health, vol. 61, no. 4, pp. 298-301, 2007.

[43] L. Bauld, K. Judge, and S. Platt, "Assessing the impact of smoking cessation services on reducing health inequalities in England: observational study," Tobacco Control, vol. 16, no. 6, pp. 400404, 2007.

[44] Royal College of Physicians, Harm Reduction in Nicotine Addiction. Helping People Who Can't Quit. A Report by the Tobacco Advisory Group of the Royal College of Physicians, Royal College of Physicians, London, UK, 2007. 


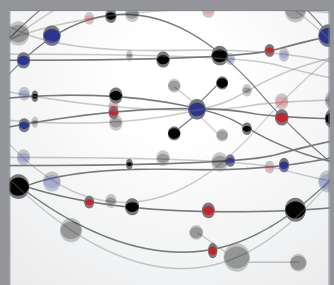

The Scientific World Journal
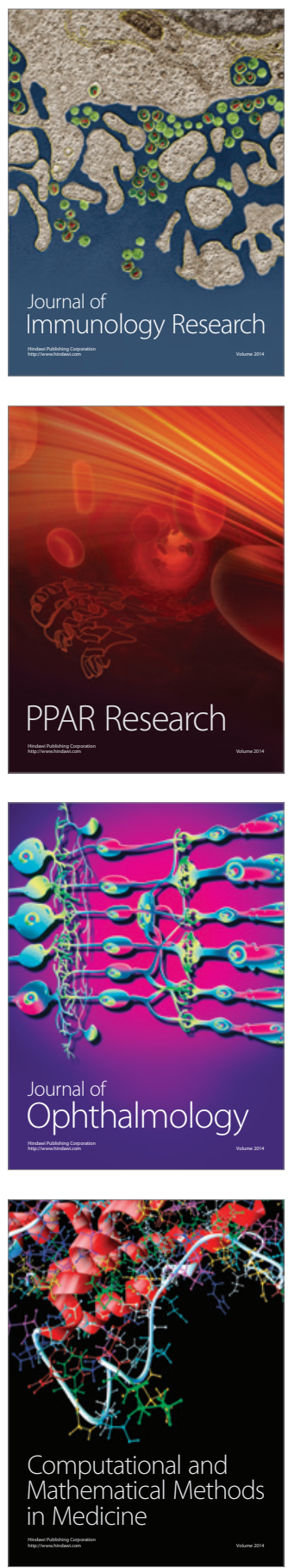

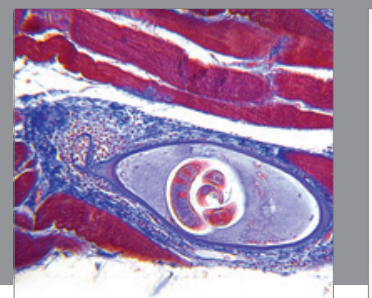

Gastroenterology

Research and Practice
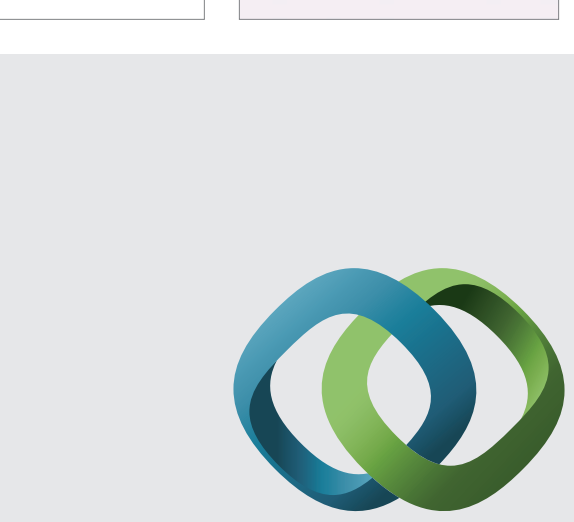

\section{Hindawi}

Submit your manuscripts at

http://www.hindawi.com
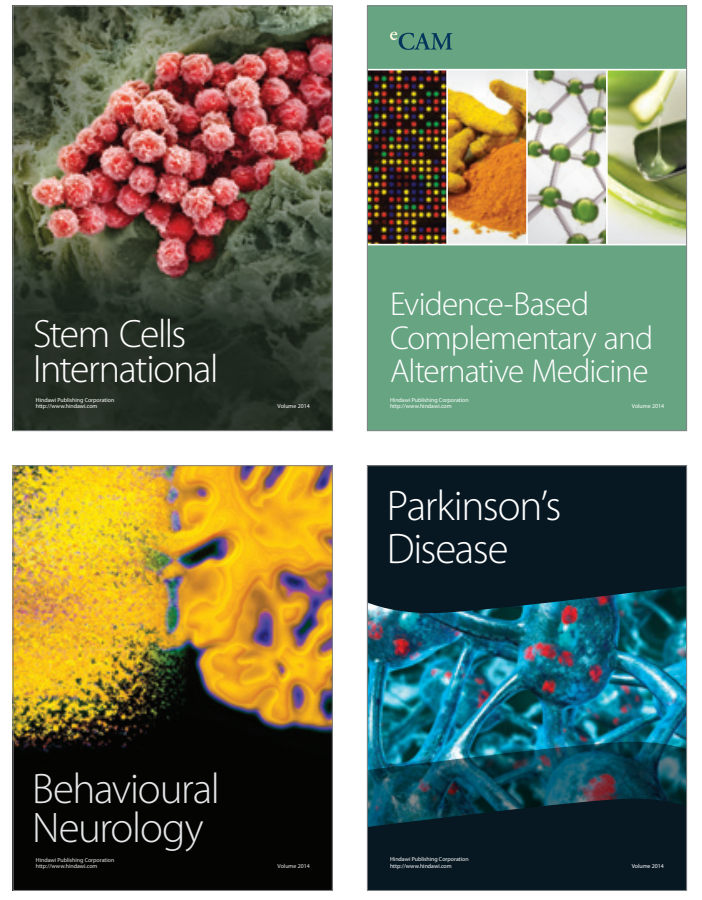
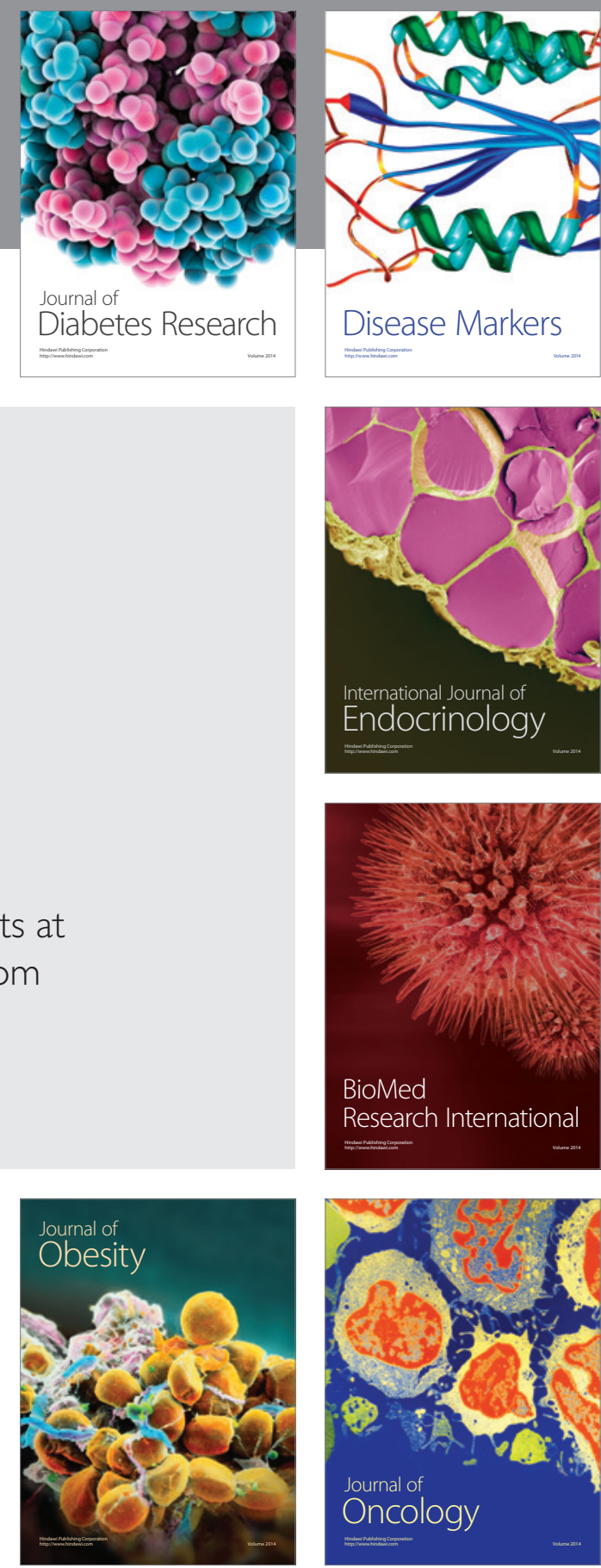

Disease Markers
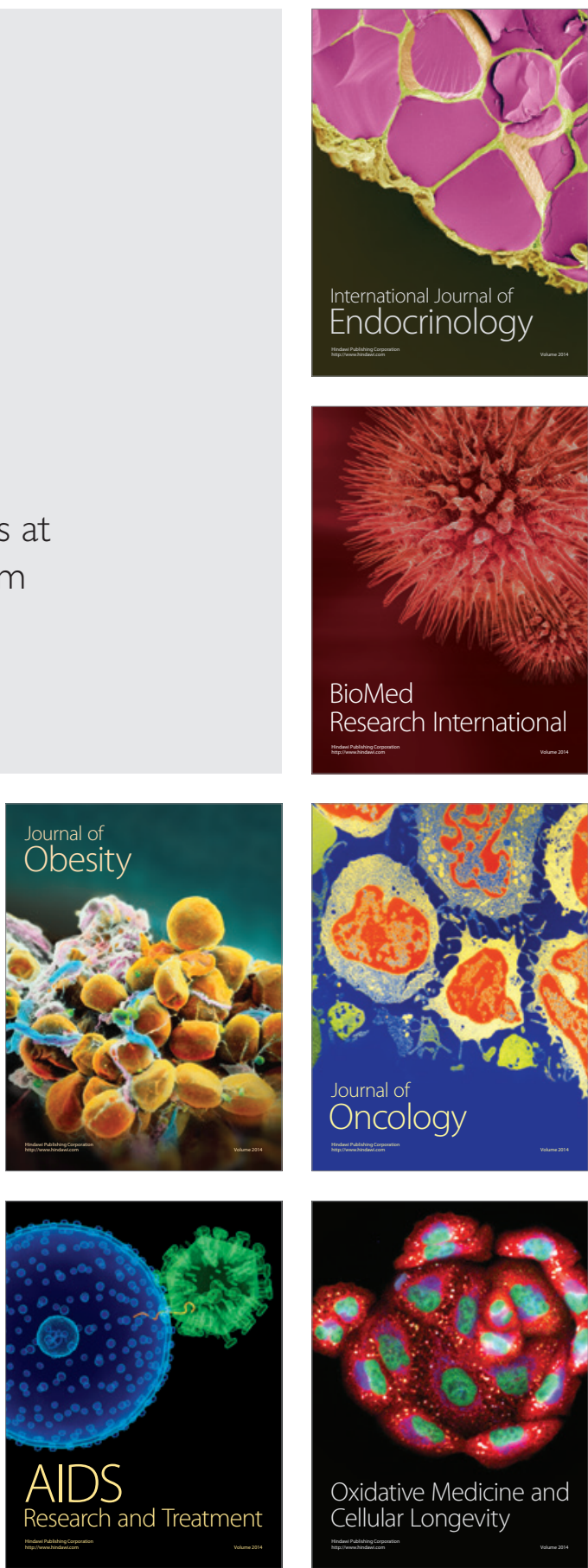